\title{
Small pharmacies are more likely to dispense antibiotics without a medical prescription than large pharmacies in Catalonia, Spain
}

C Llor (carles.llor@urv.cat) ${ }^{1}$, D L Monnet ${ }^{2}$, J M Cots ${ }^{3}$

1. Primary Healthcare Centre Jaume I, Tarragona, Spain

2. Scientific Advice Unit, European Centre for Disease Prevention and Control, Stockholm, Sweden

3. Primary Healthcare Centre La Marina, Barcelona, Spain

Citation style for this article:

Llor C, Monnet DL, Cots JM. Small pharmacies are more likely to dispense antibiotics without a medical prescription than large pharmacies in Catalonia, Spain. Euro Surveill. 2010;15(32): pii=19635. Available online: http://www.eurosurveillance.org/ViewArticle.aspx?Articleld=19635

The aim of this study was to explore the relationship between pharmacy size and the likelihood of obtaining antibiotics without medical prescription at a pharmacy. In 2008 in Catalonia, two actors presented three different cases in a randomised sample of pharmacies and asked pharmacists for an antibiotic. Pharmacies were considered as small when having limited space with only one counter and a maximum of two professionals selling medicines, as medium sized with three or four attending professionals, and as large with a large selling space and more than four attending professionals. Of the 197 pharmacies visited, 88 (44.7\%) were considered as small while only $25(12.7 \%)$ were large. Antibiotics were obtained without a medical prescription in $89(45.2 \%)$ pharmacies, mainly in small pharmacies $(63.6 \%)$, followed by medium-sized pharmacies $(35.7 \%)$ and large pharmacies (12\%) (p<0.001). Large pharmacies, that probably have a greater income, more closely followed the prevailing legislation of not selling antibiotics to patients without a medical prescription. This observation should now be confirmed in other countries where over-the-counter sales of antibiotics are prevalent and should be taken into account by programmes aiming at achieving a more prudent use of antibiotics.

\section{Introduction}

The majority of antibiotic prescriptions are used for outpatients in the community [1]. Use of antibiotics can contribute to the emergence and selection of antimicrobial-resistant bacterial strains, which have become a problem in most countries $[2,3]$. Not surprisingly, there is a relationship between the overall uptake of antibiotics in a population and resistance to antibiotics in bacteria responsible for infections in this population [4-6]. This is the background for public awareness campaigns aiming at decreasing unnecessary use of antibiotics in the community $[7,8]$.

Despite being designated as prescription-only medicines, antibiotics may be requested by and dispensed to patients without a medical prescription in many areas of the world. This has been described as common practice in developing countries [9]. In Europe, obtaining antibiotics without a medical prescription is reportedly more prevalent in Mediterranean countries than in northern countries [10-12]. In Spain, over-the-counter sale of antibiotics still occurs despite being illegal, as in other European Union countries, and despite many public awareness campaigns targeting self-medication carried out by the Ministry of Health. A prospective study was performed in 2008 in a randomised sample of pharmacies in Catalonia, Spain, with the objective of quantifying the percentage of pharmacies selling antibiotics without an official medical prescription [13]. Antibiotics were obtained in nearly $80 \%$ for a supposed urinary tract infection, and in $35 \%$ for sore throat. From our experience, certain pharmacies in Spain sell antibiotics without a prescription while others seem to scrupulously follow the law. An analysis of European data showed a relationship between the number of inhabitants per pharmacy in a country and the annual revenue from prescription medicines per pharmacy in this country [14], thus suggesting that pharmacies serving fewer inhabitants may have lower revenues from prescription medicines and an incentive for over-the-counter sales of prescription medicines. Using the same database as the prospective study mentioned above [13], the present study was performed to explore the relationship between the size of a pharmacy and the likelihood of obtaining antibiotics without a medical prescription.

\section{Methods}

The methodology of the study has been described elsewhere [13]. In brief, a prospective study was performed in 2008 in a randomised sample of 197 pharmacies in Catalonia, stratified by provinces, socioeconomic status and location. Actors impersonated two patients in three different case scenarios: sore throat, acute bronchitis, and urinary tract infection. They entered the pharmacies stating that they were not from the neighbourhood or city, that they were commercial representatives, and that they were passing through. Three levels of demand were used to convince the 
pharmacists to sell an antibiotic: Initially the actor asked for something to alleviate the symptoms of the infection. If an antibiotic was not offered, the actor said "This medication is not very strong, can't you give me something stronger?" The highest grade of demand was asking openly for an antibiotic, if the medicine was not offered by the pharmacist before.

For the present study, pharmacies were classified in three groups depending on their size (based on the actors' assessment): small pharmacies with limited space with only one counter and a maximum of two professionals selling medicines, medium pharmacies with three or four attending professionals, and large pharmacies with a large selling space and more than four attending professionals. The following variables were also considered: age of the pharmacist or salesperson attending the patient (estimated by the actor as a categorical variable: under 30 years of age, between 30 and 50 years and over 50 years), gender of the pharmacist or salesperson, location (rural or urban) and socioeconomic status of the area on the basis of the gross disposable household income per inhabitant (rich when this measure in relation to the national average was $>100$ and poor when it was (100) taken from the last official report published by the government of Catalonia [15].

We performed descriptive statistical analyses of the results using chi-square tests to compare qualitative variables and analysis of variance to compare three mean values. Differences were considered significant with $\mathrm{p}$ values under 0.05 .

\section{Results}

Of the 197 pharmacies visited, 88 (44.7\%) were considered as small while only 25 (12.7\%) were large. A total of 94 pharmacies were located in richer socioeconomical areas (47.7\%). From a demographic point of view, most Catalans live in urban areas and only 44 (22.3\%) visited pharmacies were in rural areas. Most of the pharmacies in rural areas were small (59.1\%), whereas most of the pharmacies located in urban areas were mediumsized $(46.4 \%)$, regardless of the socioeconomic status. Because the sample of pharmacies was stratified by provinces, socioeconomic status and location, but not by size, the urinary tract infection scenario was used by the actors mostly in small pharmacies, while a higher percentage of sore throat and acute bronchitis scenarios were used in medium-sized and large pharmacies ( $p<0.05)$.

Antibiotics were obtained without a medical prescription in $89(45.2 \%)$ of the 197 pharmacies surveyed. Antibiotics were obtained from $79.7 \%$ of the pharmacies when a urinary tract infection was simulated, from $34.8 \%$ for a sore throat and from $16.9 \%$ for an acute bronchitis. Sales of antibiotics without a medical prescription were highest in small pharmacies (63.6\%) and lowest in large pharmacies (12\%) (p<0.001) (Table). This pattern was found in all three simulated case scenarios. In the few large pharmacies in rural areas, antibiotics were never obtained.

The percentage of antibiotics sold was slightly higher among community pharmacies in poorer areas $(49.5 \%)$ compared with those located in richer areas (40.4\%), with no statistically significant difference. Overall, no

\section{TABLE}

Percentage of pharmacies in which the antibiotic was obtained, by simulated case of infection, size of pharmacy and type of area, Catalonia, $2008(\mathrm{n}=197)$

\begin{tabular}{|c|c|c|c|c|c|}
\hline \multirow{2}{*}{$\begin{array}{l}\text { Simulated case of infection } \\
\text { by type of area }\end{array}$} & \multirow{2}{*}{$\begin{array}{c}\text { All pharmacies } \\
n(\%)\end{array}$} & \multicolumn{3}{|c|}{$\begin{array}{c}\text { By size of pharmacy } \\
n(\%)\end{array}$} & \multirow[b]{2}{*}{$\mathrm{p}$} \\
\hline & & Small pharmacies & $\begin{array}{l}\text { Medium-sized } \\
\text { pharmacies }\end{array}$ & Large pharmacies & \\
\hline \multicolumn{6}{|l|}{ Urban } \\
\hline Urinary tract infection & $41 / 52(78.8)$ & $23 / 25(92.0)$ & $16 / 24(66.7)$ & $2 / 3(66.7)$ & NS \\
\hline Sore throat & $20 / 53(37.7)$ & $15 / 23(65.2)$ & 4/18 (22.2) & $1 / 12(8.3)$ & $<0.01$ \\
\hline Acute bronchitis & $10 / 48(20.8)$ & $6 / 14(42.9)$ & $4 / 29(13.8)$ & $0 / 5(0)$ & $<0.05$ \\
\hline All cases & $71 / 153(46.4)$ & $44 / 62(71.0)$ & $24 / 71(33.8)$ & $3 / 20(15.0)$ & $<0.001$ \\
\hline \multicolumn{6}{|l|}{ Rural } \\
\hline Urinary tract infection & $14 / 17(82.4)$ & 9/10 (90.0) & $5 / 6(83.3)$ & $0 / 1(0)$ & NS \\
\hline Sore throat & $4 / 16(25.0)$ & $3 / 12(25.0)$ & $1 / 3(33.3)$ & $0 / 1(0)$ & NS \\
\hline Acute bronchitis & $0 / 11(0)$ & $0 / 4(0)$ & $0 / 4(0)$ & $0 / 3(0)$ & NS \\
\hline All cases & $18 / 44(40.9)$ & $12 / 26(46.2)$ & $6 / 13(46.2)$ & $0 / 5(0)$ & NS \\
\hline \multicolumn{6}{|l|}{ Both areas combined } \\
\hline Urinary tract infection & $55 / 69(79.7)$ & $32 / 35(91.4)$ & $21 / 30(70.0)$ & $2 / 4(50.0)$ & $<0.05$ \\
\hline Sore throat & $24 / 69(34.8)$ & $18 / 35(50.7)$ & $5 / 21(23.8)$ & $1 / 13(7.7)$ & $<0.01$ \\
\hline Acute bronchitis & $10 / 59(16.9)$ & 6/18 (33.3) & 4/33(12.1) & $0 / 8(0)$ & $<0.05$ \\
\hline All cases & $89 / 197(45.2)$ & $56 / 88(63.6)$ & $30 / 84(35.7)$ & $3 / 25(12.0)$ & $<0.001$ \\
\hline
\end{tabular}

NS: non significant $(p \geq 0.05)$ 
differences were observed in the sales of antibiotics between urban and rural areas $(46.5 \%$ versus $40.9 \%$, non-significant). When performing sub-analyses by pharmacy size, this pattern was observed for large and small pharmacies, but curiously not for pharmacies considered of medium size (Figure). For medium-sized pharmacies, the sale of antibiotics without prescription was slightly greater in rural compared to urban areas ( $46.2 \%$ vs. $33.8 \%$ ), although no statistical difference was found. Nor did we find any statistical differences in the sales of antibiotics without a medical prescription with regard to the gender of the actor or to the age or gender of the pharmacist or salesperson, although a trend towards selling more antibiotics was observed among men and those over the age of 50 years.

\section{Discussion}

Even though pharmacists are not allowed to dispense prescription-bound medicines without a medical prescription under any circumstance, antibiotics were obtained without a medical prescription in nearly one half of the visited community pharmacies. The results of this study confirm the initial hypothesis that small pharmacies are more likely to sell antibiotics without a prescription than large pharmacies. One reason may be that small pharmacies have a smaller income than large pharmacies, which may be an incentive to generate more revenues by selling prescription-bound medicines over-the-counter, without a medical prescription. In addition, in small pharmacies, the person behind the counter may be the owner, whereas in large pharmacies the person behind the counter is more likely to be a pharmacist or a salesperson who is an employee who has no direct benefit from selling more medicines. To our knowledge, this is the first study to investigate the relationship between size of a pharmacy and over-thecounter sale of antibiotics. In a general study of sales of medications without medical prescription, Rogers et al. observed that the lowest level of over-the-counter medicine purchases was recorded in inner cities whilst the highest levels were found in rural pharmacies and small towns [16]. In our study, more than $80 \%$ of the

\section{FIGURE}

Percentage of pharmacies in which the antibiotic was obtained depending on size of pharmacy and type of area, Catalonia, 2008 ( $\mathrm{n}=197)$

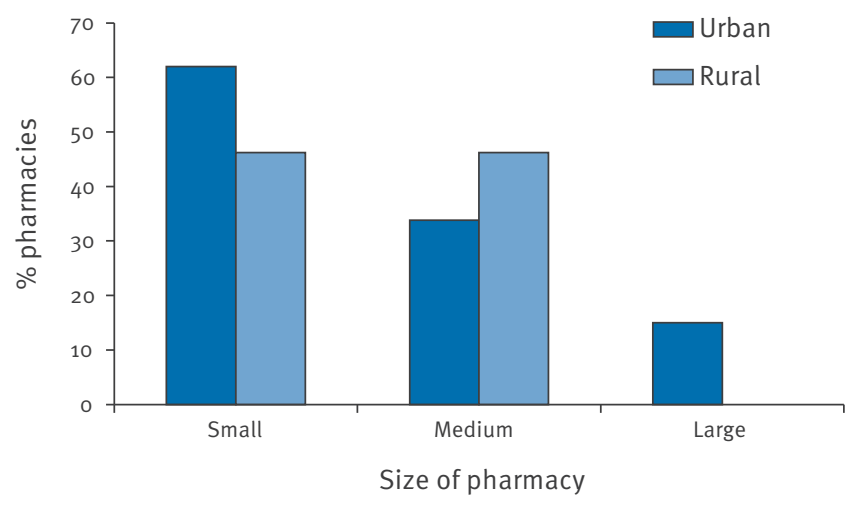

large pharmacies were located in cities and these pharmacies were more likely to follow the legislation.

The actors who participated in the study had many years of experience acting as patients in university tests for medical students and trainees in the specialty of family medicine. Nevertheless, this study has some limitations. Firstly, we did not obtain information on the income of the visited pharmacies. As a surrogate, we classified pharmacies according to their size based on the number of salespersons attending customers. These parameters were observed by the two actors. Another limitation is that we could not study the relationship between the profession, pharmacist or other employee, of the attending person and the likelihood of selling an antibiotic without a medical prescription. The actors attempted to approach them as normal customers and asking about the profession of the attending person may have raised suspicion. Another limitation to be taken into account is the fact that the distribution of cases was not uniform among the different sizes of pharmacies because the actors simulated cases with urinary tract infections less frequently and cases with sore throat more frequently in the largest pharmacies, while cases of acute bronchitis were simulated more frequently the medium-sized pharmacies. However, sales of antibiotics were highest among small pharmacies and lowest in large pharmacies, and this pattern was found for all three case scenarios.

With the growing importance of self care, deregulation of medicines and the perceived need to reduce workload of general practitioners, community pharmacists have, in the last years, sought to establish themselves as advice givers about medicine usage and symptom management [17]. In addition, pharmacists are very accessible in Spain and reach nearly the entire population, with a mean of 2,143 inhabitants served by each pharmacy [18]. This is one of the lowest ratios observed in the European Union. However, Spain is one of the European countries where community pharmacies are more strictly regulated, with clear restrictions on ownership. For instance only pharmacists are allowed to own a pharmacy and each pharmacist can only own a maximum of one pharmacy. As a consequence, pharmacy chains are not allowed in Spain [18]. Despite strict regulations, many pharmacies still sell antibiotics to patients without a medical prescription. This practice is also common, although illegal, in at least one other European country [12], and might be present in other countries since self-medication with antibiotics is common in southern and eastern European countries [19]. Most patients are aware of this practice and go directly to a pharmacy rather than wait and pay for a medical consultation. The practice is also well known from immigrants from countries that are strictly regulated in this context. Väänänen et al., for example, showed that the antibiotic use habits of Finns change completely when they move to Spain [20]. 
This study showed that small pharmacies in Catalonia are more likely to dispense antibiotics without a medical prescription, whereas large pharmacies, that probably have a greater income, more closely follow the prevailing legislation of not selling antibiotics to patients without a medical prescription. This observation should now be confirmed in other countries where over-the-counter sales of antibiotics are prevalent and should be taken into account by programmes aiming at achieving a more prudent use of antibiotics.

\section{Acknowledgements}

We wish to thank the actors who participated in this study for their valuable contribution to the field work of this study.
17. Turner P. The Nuffield Report: a signpost for pharmacy. Br Med J (Clin Res Ed). 1986;292(6527):1031-3.

18. Lluch M, Kanavos P. Impact of regulation of community pharmacies on efficiency, access and equity. Evidence from the UK and Spain. Health Policy. 2010;95(2-3):245-54.

19. Grigoryan L, Burgerhof JG, Degener JE, Deschepper R, Lundborg CS, Monnet DL, et al. Self-Medication with Antibiotics and Resistance (SAR) Consortium. Determinants of self-medication with antibiotics in Europe: the impact of beliefs, country wealth and the healthcare system. J Antimicrob Chemother. 2008;61(5):1172-9.

20. Väänänen $M H$, Pietilä K, Airaksinen M. Self-medication with antibiotics- Does it really happen in Europe? Health Policy. 2006;77(2):166-71.

\section{References}

1. Carbon C, Bax RP. Regulating the use of antibiotics in the community. BMJ. 1998;317(7159):663-5.

2. Hawkey PM. Action against antibiotic resistance; no time to lose. Lancet. 1998;351(9112):1298-9.

3. World Health Organization (WHO). Report on infectious diseases 2000: overcoming antibacterial resistance. Switzerland: WHO; 2000. Available from: http://www.who.int/ infectious-dissease-report/2000/index.html

4. Austin DJ, Kristinsson KG, Anderson RM. The relationship between the volume of antimicrobial consumption in human communities and the frequency of resistance. Proc Natl Acad Sci U S A. 1999;96(3):1152-6.

5. Goossens H, Ferech $M$, Vander Stichele R, Elseviers $M$, the ESAC Project Group. Outpatient antibiotic use in Europe and association with resistance: a cross-national database study. Lancet. 2005;365(9459):579-87.

6. Steinke D, Davey P. Association between antibiotic resistance and community prescribing: a critical review of bias and confounding in published studies. Clin Infect Dis. 2001;33 Suppl 3:S193-205.

7. Earnshaw S, Monnet DL, Duncan B, O'Toole J, Ekdahl K, Goossens H, et al. European Antibiotic Awareness Day, 2008 - the first Europe-wide public information campaign on prudent antibiotic use: methods and survey of activities in participating countries. Euro Surveill. 2009;14(30):pii=19280. Available from: http://www.eurosurveillance.org/ViewArticle. aspx?Articleld $=19280$

8. Goossens H, Guillemot D, Ferech M, Schlemmer B, Costers M, van Breda $M$, et al. National campaigns to improve antibiotic use. Eur J Clin Pharmacol. 2006;62(5):373-

9. Hart CA, Kariuki S. Antimicrobial resistance in developing countries. BMJ. 1998;317(7159):647-50.

10. European Commission. [Antibiotics]. Eurobaromètre Spécial 183-3/Vague 58.2. European Opinion Research Group EEIG]. Luxembourg: 2003. [French]. Available from: http://ec.europa. eu/health/ph_threats/com/mic_res/eb582_antibio_fr.pdf

11. Grigoryan L, Haaijer-Ruskamp FM, Burgerhof JG, Mechtler R, Deschepper R, Tambic-Andrasevic A, et al. Self-medication with antimicrobial drugs in Europe. Emerg Infect Dis. 2006;12(3):452-9.

12. Plachouras D, Kavatha D, Antoniadou A, Giannitsioti E, Poulakou G, Kanellakopoulou K, et al. Dispensing of antibiotics without prescription in Greece, 2008: another link in the antibiotic resistance chain. Euro Surveill. 2010;15(7):pii=19488. Available from: http://www.eurosurveillance.org/ViewArticle. aspx?Articleld $=19488$

13. Llor C, Cots JM. The sale of antibiotics without prescription in pharmacies in Catalonia, Spain. Clin Infect Dis. 2009;48(10):1345-9.

14. Monnet DL. How do people obtain antibiotics in European countries: an overview. Available from: http://www.santesports.gouv.fr/dossiers/antibiotiques/Pres_Monnet.pdf

15. Official statistics website of Catalonia. Gross disposable household income per inhabitant, 2000-2004. Counties and areas. Institut d'Estadística de Catalunya. Barcelona. Available from: http://www.idescat.cat/pub/?id=aec\&n=370\&lang=en

16. Rogers A, Hassell K, Noyce P, Harris J. Advice-giving in community pharmacy: variations between pharmacies in different locations. Health Place. 1998;4(4):365-73. 